\title{
Discriminative Multi-Layer Illumination-Robust Feature Extraction for Face Recognition ${ }^{\text {th }}$
}

\author{
Yu-Feng Yu', Dao-Qing Dai ${ }^{\mathrm{a}, *}$, Chuan-Xian Ren ${ }^{\mathrm{a}}$, Ke-Kun Huang ${ }^{\mathrm{b}, \mathrm{a}}$ \\ ${ }^{a}$ Intelligent Data Center and Department of Mathematics, Sun Yat-Sen University, Guangzhou \\ 510275, China. \\ ${ }^{b}$ School of Mathematics, JiaYing University, Meizhou, Guangdong, 514015, China.
}

\begin{abstract}
Tackling illumination variation is a major problem and it is also an important challenge for practical face recognition systems. Some related methods consider that lighting intensity components mainly lie in large-scale features, and they use a lot of image decomposition techniques to extract the small-scale illuminationinvariant features and remove the large-scale features from original face images. However, it argues that the large-scale features contain a lot useful information which can be further extracted, and the small-scale illumination-invariant features are not robust enough due to they contain some detrimental features (noise, etc.). In this paper, we propose a discriminative multi-layer illumination-robust feature extraction (DMI) model to address this problem. First, we decompose the large-

\footnotetext{
This work is supported in part by the National Science Foundation of China under Grants 11631015, 61203248, 11171354, 90920007, 61375033, 61403164 and 61572536. in part by the Ministry of Education of China under Grant SRFDP-20120171120007 and Grant 20120171110016, in part by the Natural Science Foundation of Guangdong Province under Grant S2013020012796, in part by the Fundamental Research Funds for the Central Universities under Grant 161gzd16.

*Corresponding author

Email addresses: yuyufeng220@163.com (Yu-Feng Yu), st sddq@mail . sysu. edu . cn (Dao-Qing Dai), rchuanx@mail.sysu.edu. cn (Chuan-Xian Ren), kkcocoon@163. com (Ke-Kun Huang)
} 
scale features into multi-layer small-scale illumination-robust features as a linear combination, and then a weight is assigned to each layer to adjust its importance and influence. The idea is to take full advantage of these useful information in large-scale features for face recognition. Second, we learn a discriminant filter to improve the robustness and statistical discriminative ability of the reconstructed illumination-robust face for face recognition under poor lighting conditions. Extensive experiments on three benchmark face databases and a video image database show that DMI performs better than the related methods, especially in difficult lighting conditions.

Keywords: Face recognition, decomposition technique, illumination-invariant feature, discriminant filter, small-scale

\section{Introduction}

$2 \quad$ Face recognition has attracted a great deal of attentions during the past sev3 eral decades. Various approaches have been proposed, including Eigenface [1],

4 Fisherface [2], Independent Component Analysis (ICA) [3], Laplacianfaces [4] 5 and some regression models [5] [6] [7] [8] [9]. They can improve recognition 6 performance to several kinds of variations. However, the performance of many 7 existing algorithms are highly sensitive to illumination variations. In recent years, 8 a variety of methods have been proposed to solve the illumination problem. The 9 predominant ones include Histogram Equalization (HE) [10] [11], Logarithmic Transform [12]. These methods use traditional image processing model to normalize illumination unbalance, which can improve visual results to some extent, but it is difficult to account for different lighting conditions.

Some methods are proposed to extract the illumination-invariant representa- 
tion of face images under varying lighting conditions. Most of them are based on Lambertian reflectance model. Lambertian reflectance model factorizes an image as a multiplication of a light intensity component and a surface albedo component. In general, a face image $I(x, y)$ is regarded as product $I(x, y)=\rho(x, y) S(x, y)$, where $\rho(x, y)$ is the surface albedo and $S(x, y)$ is the final light received at location $(x, y)$. However, it is difficult to obtain the $\rho$ value of small-scale features from real images. In [13], Wang et al. proposed the SQI model, which extracts intrinsic, illumination-invariant features from a face image based on the QI technique. However, SQI has trouble keeping sharp edges in low frequency illumination fields. In order to preserve sharp edges of images, edge detection technique has attracted a lot of attentions. Le [14] proposed a new hyperbolic distribution and hyperbolic mask for edge detection, in which edge-detection error probability as a function of the half mask size is estimated using both masks in Gaussian- and hyperbolicdistributed pixel-intensity images. The experimental results show that the hyperbolic mask is effective and noise robust for edge detection. In [15], Hegade et al. considered morphological edge detection as pre-processing technique to normalize pose and expression variations, which can improve the performance to some extent for face recognition.

Methods based on two-dimensional function decomposition are well-known and popular. For instance, Chen et al.[16] proposed the logarithm total variation (LTV) model, which utilizes the edge-preserving capability of the total variation model. LOG-DCT [17] proposed by Chen et al. by using image processing techniques to normalize the images to appear stable under different illumination conditions, they employed discrete cosine transform to compensate for illumination variations in the logarithm domain. Based on the common assumption, Gradient- 
faces (Gradf) [18] is regarded as insensitive to illumination variant and is extracted from the gradient domain. Chen et al.[19] constructed the intrinsic illumination subspaces from illumination images of intrinsic images, and then proposed a lighting normalization method. In [20], the authors proposed an illumination normalization method based on Weber's law under varying illumination, which they called Weberface (Webf) is an illumination insensitive representation. Lai et al. [21] considered that the small-scale surface albedo is not robust enough because of containing some detrimental sharp features, and proposed a MLSA algorith$\mathrm{m}$ to decompose the surface albedo as a linear combination. Recently, Inspired by the fact that normalized coefficients of the singular value decomposition are insensitive to different lighting conditions, Kim et al. [22] built an illuminationinvariant face representation method, called SVD face. In addition, the decomposition methods based on wavelets have been widely used in face recognition. Kwak et al. [23] first used the wavelet decomposition to extract intrinsic features of face images, and then combined Fisherface method and fuzzy integral into a single coherent classification platform for face recognition. Goh et al. [24] proposed wavelet based illumination invariant preprocessing (WIIP) in face recognition. They decomposed a facial image into low and high frequency components using discrete wavelet transform (DWT) decomposition and set the illumination component (low-pass component) as zero. Then both the processed illumination component (low-pass component) and the reflectance component (high-pass component) were used to perform inverse DWT. The experimental results show that these decomposition methods based on wavelets achieve salient performance for face recognition.

Image feature descriptor-based methods have been widely used in face recog- 
nition due to their great robustness. Typical one like LBP, introduced by Ojala et al. [25], has gained many attentions due to its simplicity and excellent performance for face recognition. LBP encodes each local $3 \times 3$ neighborhood structure into a binary series by thresholding its neighbor pixel intensity with the center one. Instead of binary code, Local Ternary Patterns (LTP)[26], adopted a ternary code pattern to extract illumination-invariant features. Compared with LBP, LTP is less sensitive to noise in uniform regions. Lei et al. [27] focused on LBP-like feature extraction and proposed a novel discriminant face descriptor (DFD), in which the difference of the features between images of the same person are minimized and that between images from different people are maximized. Different from LBP-based methods, Ren et al. [28] considered that Gabor features in each band can be combined by a vector, and proposed a band-reweighed Gabor kernel embedding algorithm to deal with face recognition with illumination or pose variation. Other feature extraction-based methods such as Local Directional Patterns (LDP) [29], Enhanced LDP [30], Local Directional Number Patterns (LDN) [31], AHELDP [32], WFSDMS [33] also have gained much attention because of their robustness to several kinds of variations, but they still have some room to improve the performance when illumination variation dominates the main variation.

It can be seen that LTV, LOG-DCT, Graf and Webf conclude that light intensity mainly lies in large-scale component of an image. Although these methods can obtain good results to some extent, they are unrobust to reference images under difficult lighting conditions and sensitive to some sharp detrimental noise pixels (e.g. shadow casts by big facial organs, shadow borders). In the field of signal processing, noise usually exists in the small-scale features. This is why many noise robust models [34] [35] have been proposed to overcome this problem. In 


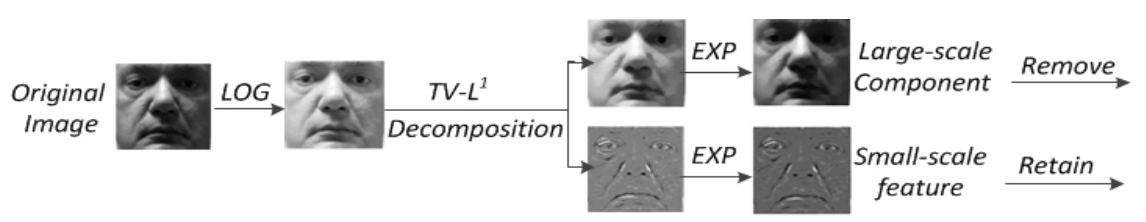

(a) LTV

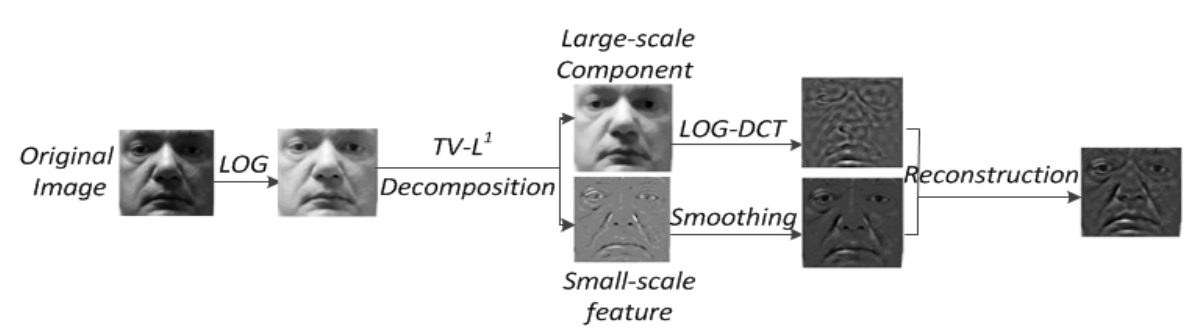

(b) $\mathrm{S} \& \mathrm{~L}(\mathrm{LOG}-\mathrm{DCT})$

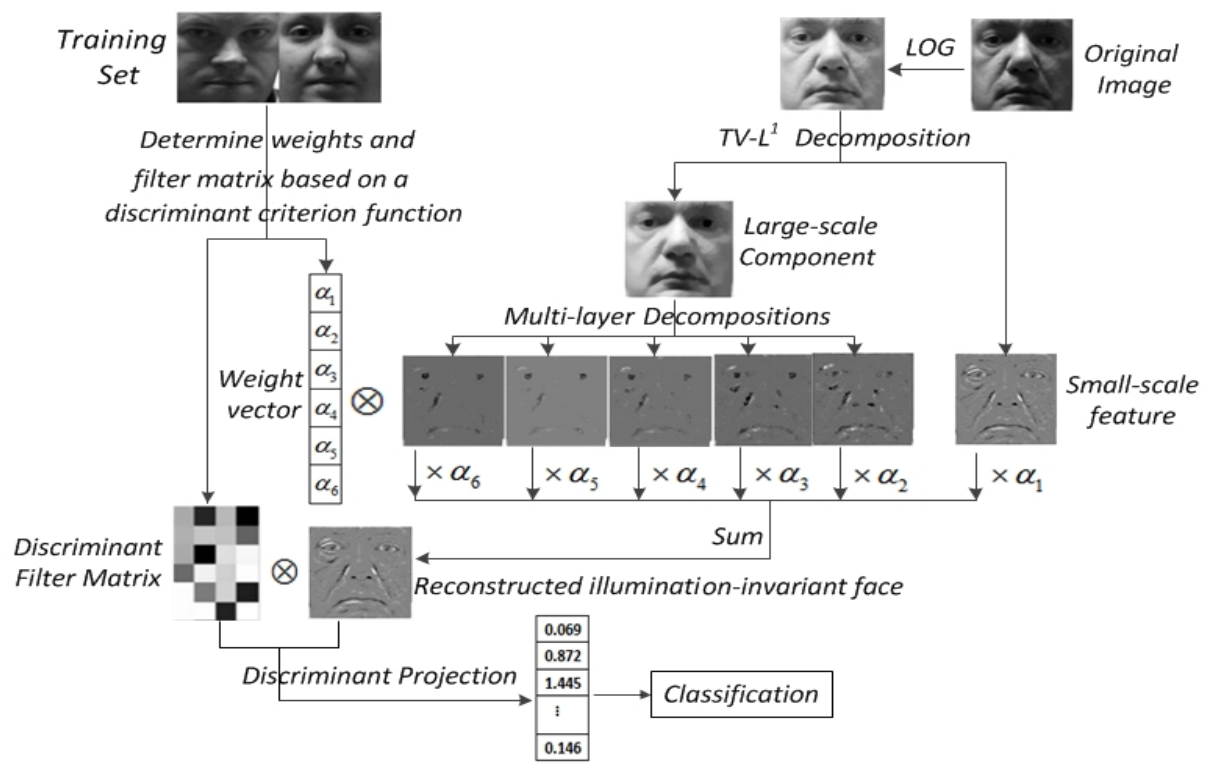

(c) DMI

Figure 1: Comparisons between schemes of LTV, S\&L and DMI. (a): LTV retains small-scale component and removes large-scale component. (b): S\&L combines smoothed small-scale component and normalized large-scale component. (c): DMI retains small-scale component and decomposes large-scale component into multi-layer illumination-robust features, and then takes them as a linear combination. 
addition, these existing methods still lack statistical discriminant face information. Hence, the performance is unstable in complicated situations when lighting conditions are extremely poor.

In this paper, we propose an illumination-robust feature extraction method with statistical discriminant face information named discriminative multi-layer illumination-robust feature extraction (DMI) for face recognition under poor lighting conditions. The flowchart of our proposed method is shown in Figure. 1c. It is worth mentioning that the proposed DMI is different from other illuminationinvariant feature extraction methods [13][16][21]. First, these methods only retain small-scale component and remove large-scale component, while we argue that large-scale component contains useful information for face recognition, which can be further extracted, instead of discarded. Second, they do not learn discriminant filter, while DMI learns a discriminative filter to remove noise from the reconstructed illumination-robust face image and improve the statistical discriminative ability. In addition, S\&L [36] also argues that large-scale component is useful for recognition. But it only uses LOG-DCT algorithm to preprocess the large-scale component, which is difficult to separate the extrinsic illumination variations and intrinsic facial from large-scale component. We summarize the favorable properties and main contributions of DMI as follows.

1. Proposing a multi-layer decomposition algorithm. It decomposes the largescale component as a linear combination of several detailed layers and can extract different kinds of beneficial and illumination-robust features in different layers, which are ignored by traditional illumination-treating methods (e.g. LTV, LOGDCT, etc).

2. Introducing a weighting strategy. Since each illumination-robust feature 
layer contains different discriminant information for face recognition. A weight is allocated to each layer to control the importance and influence of this layer. A linear sum of all the weighted layers form the reconstructed illumination-robust face image. The weight in each layer can be integrated into within-class scatter matrix and between-class scatter matrix of small-scale illumination-robust features.

3. Introducing a discriminative filter. Considering that noise usually exist$\mathrm{s}$ in the small-scale illumination-robust features, our method learns a discriminant filter to remove noise from the reconstructed illumination-robust face and improve the statistical discriminative ability for face recognition under poor lighting conditions. Similarly, the learned discriminant filter can also be integrated into within-class scatter matrix and between-class scatter matrix of small-scale illumination-robust features.

4. Proposing an illumination-robust face recognition system. We develop a discriminant criterion function and propose an effective DMI algorithm to determine all the weights and the discriminant filter matrix simultaneously.

The rest of the paper is organized as follows. In section 2, we propose the DMI model and develop a discriminant criterion function to select the suitable layer weights and the discriminant filter matrix. Section 3 reports experimental results on benchmark databases, followed by the conclusion in section 4 .

\section{Discriminative multi-layer illumination-robust features extraction}

In this section, each step of the proposed method is described in detail. In section 2.1, we first introduce the theoretical background, and we further discuss the motivation in section 2.2. Then the weighted-layer small-scale illumination-robust feature extraction method is proposed in section 2.3. In section 2.4, a discriminant 
filter learning method is proposed. In order to determine all the layer weights and the discriminant filter matrix simultaneously, we propose an effective DMI algorithm based on a statistical discriminant Fisher criterion function in section 2.5. In section 2.6, we take a comparison analysis between the proposed DMI and the related works.

\subsection{Theoretical Background}

We first introduce the reflectance model, which is the theoretical basis of removing varying illumination for face images. Then we discuss the Logarithmic Total Variation (LTV) [16] model, which serves as the basis of our proposed method.

The reflectance model defines the pixel value as a production of reflectance and illumination component as follows:

$$
I(x, y)=R(x, y) L(x, y)
$$

where $I(x, y)$ is face image pixel value, $R(x, y)$ and $L(x, y)$ denote the reflectance component and illumination effect at each point $(x, y)$, respectively. Here, $R(x, y)$ only depends on the characteristics of the surface of the object, so it can be regarded as intrinsic representation of a face image and contains illumination invariant features. The objective is to separate the reflectance component $R$ and the illumination effect $L$ from real image for face recognition.

Inspired by the SQI, Chen et al.[16] proposed LTV model, which makes use of the edge preservation and multi-scale decomposition capability of the TV- $L^{1}$ [37] model. In LTV model, based on reflectance model, the following decomposition 
is obtained:

$$
\begin{aligned}
I(x, y) & =R(x, y) L(x, y) \\
& =\left(\frac{R(x, y)}{\rho_{l}(x, y)}\right)\left(\rho_{l}(x, y) L(x, y)\right) \\
& =\rho(x, y) S(x, y),
\end{aligned}
$$

where $\rho_{l}(x, y)$ denotes the albedo of large-scale skin areas and background. $\rho(x, y)$ is the surface albedo, which only contains the small intrinsic facial structures and $S(x, y)$ is the final light received at location $(x, y)$, which contains large intrinsic structures as well as extrinsic illumination.

The objective of LTV is to retain the small intrinsic facial structure $\rho$ and remove the large-scale component $S$. To apply the additive TV- $L^{1}$ model to the multiplicative model, LTV takes a logarithm transform on both sides of (2):

$$
\begin{aligned}
f(x, y) & =\log I(x, y) \\
& =\log \rho(x, y)+\log S(x, y) \\
& =v+u .
\end{aligned}
$$

Then applying the TV- $L^{1}$ model, $f(x, y)$ is decomposed as follows:

$$
\begin{gathered}
\hat{u}=\arg \min _{u} \int_{\hat{v}=f-\hat{u},}|\nabla u|+\lambda|f-u| d x, \\
\hat{v},
\end{gathered}
$$

where $\int|\nabla u|$ is the total variation of $u$, scalar threshold $\lambda$ controls the selection of features with unknown intensity values. After getting $\hat{v}$, exponent transform is performed on it to finally get the small-scale surface albedo $\rho$ as follows:

$$
\rho(x, y)=\exp (\hat{v})
$$

Figure. 1a illustrates the pipeline of the LTV method. 


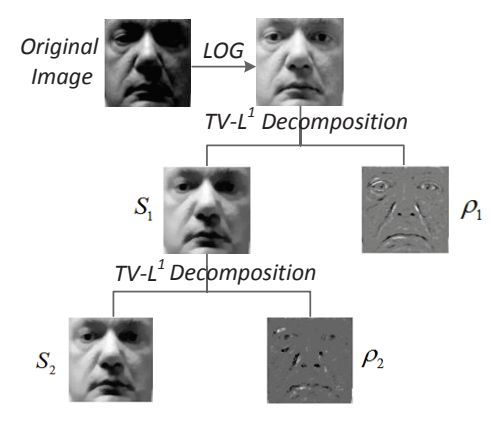

Figure 2: The extraction process of the small-scale illumination-invariant features $\rho_{1}$ and $\rho_{2}$. In the first experiment, $\rho_{1}$ is used as facial features for recognition. In the second experiment, we regard $\rho_{1}+\rho_{2}$ as facial features for recognition. Experimental results are provided in Table 1 .

\subsection{Motivation}

Many existing methods attempt to separate the reflectance component and illumination effect from real images, unfortunately, estimating $\rho$ or $S$ from $I$ is an ill-posed problem [38]. To address this problem, LTV utilizes the multi-scale decomposition ability of the TV- $L^{1}$ model to extract the small-scale feature $\rho$. It is effective to some extent, but many noise exist in the small-scale features, which leads that the small-scale feature $\rho$ is not robust enough. What's more, the large-scale component $S$ is removed by LTV, containing not only extrinsic illumination and shadow borders, but also some intrinsic facial structures (illuminationinvariant features), which are beneficial for face recognition. This implies that these illumination-invariant features can be further extracted from the large-scale component $S$. To verify this point, we conduct two experiments of face recognition using the Extended Yale B database (The detailed description of the database can be seen in section 3.1). The Subset 1 is used for training, the other 4 Subsets are used for testing, respectively. All the images from the Extended Yale B 
Table 1: Face recognition on the Extended Yale B database use $\rho_{1}$ and $\rho_{1}+\rho_{2}$, respectively.

\begin{tabular}{ccccc}
\hline Features & Subset 2 & Subset 3 & Subset 4 & Subset 5 \\
\hline$\rho_{1}$ & 99.12 & 96.71 & 86.28 & 89.66 \\
$\rho_{1}+\rho_{2}$ & 99.56 & 97.81 & 91.92 & 92.86 \\
\hline
\end{tabular}

database are decomposed into small-scale component $\rho_{1}$ and large-scale component $S_{1}$. In the first experiment, only the small-scale component $\rho_{1}$ is directly used as facial features for recognition. In the second experiment, we decompose the large-scale component $S_{1}$ into small-scale illumination-invariant feature $\rho_{2}$ and large-scale light intensity component $S_{2}$ via the TV- $L^{1}$ model again. Then we regard the linear combination of small-scale illumination-invariant features $\rho_{1}$ and $\rho_{2}$ as facial features for recognition. Figure. 2 illustrates how the small-scale illumination-invariant features $\rho_{1}$ and $\rho_{2}$ are extracted, and the recognition results are shown in Table 1. As shown, we can find that the recognition rates of $99.12 \%$, $96.71 \%, 86.28 \%$ and $89.66 \%$ are got from Subset 2 to Subset 5, respectively in the first experiment. In the second experiment, we use the linear combination of small-scale illumination-invariant features $\rho_{1}$ and $\rho_{2}$ as facial features for recognition, the recognition rates of $99.56 \%, 97.81 \%, 91.92 \%$ and $92.86 \%$ are obtained from Subset 2 to Subset 5, respectively. It is clear that the $\rho_{1}+\rho_{2}$ achieves higher recognition rates than $\rho_{1}$. The superiority of $\rho_{1}+\rho_{2}$ is getting more significant than $\rho_{1}$ when lighting conditions are getting worse from Subset 3 to Subset 5 .

From the experimental results we have the conclusions that: 1) The large-scale component $S_{1}$ contains a lot of useful information for face recognition, and these information (small-scale illumination-invariant features) as facial features should be further extracted, instead of discarded. 2) The linear combination of multi-layer 
small-scale features help to get higher recognition rates for images with extreme illumination changes. Therefore, taking full advantage of these information is very important for face recognition. Based on the multi-scale decomposition, we can further decompose the large-scale component and reconstruct illuminationrobust face image.

\subsection{Weighted-layer small-scale illumination-robust features extraction}

In this subsection, we first demonstrate how a face image can be decomposed into multi-layer small-scale illumination-robust features and large-scale lighting intensity component. Based on LTV [16] model, a face image $I$ can be decomposed into $l$ layers of different small-scale illumination-robust features as Algorithm 1:

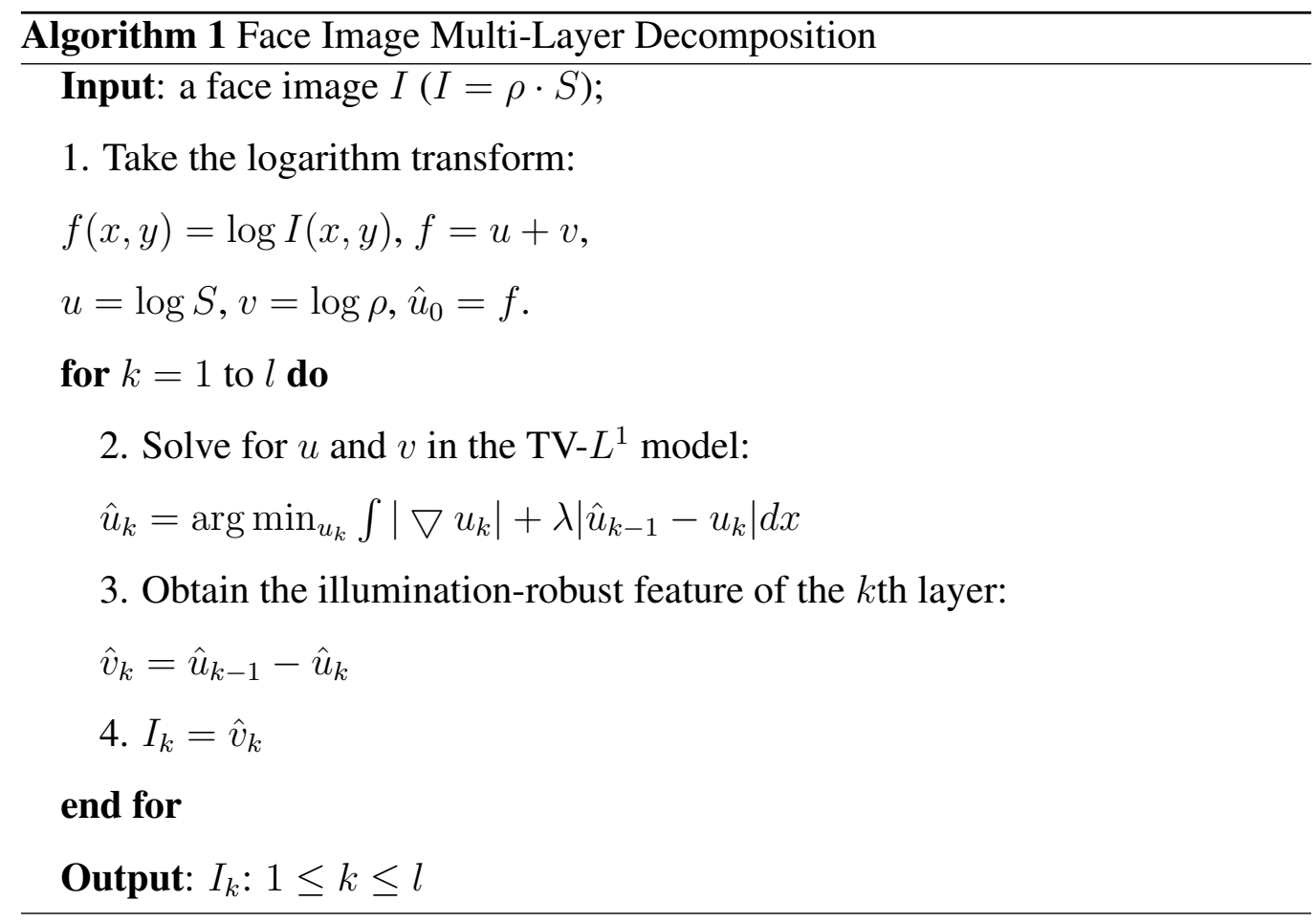


Since each small-scale illumination-robust feature layer $\left(I_{k}\right)$ contains different discriminant information for face recognition, we assign a weight to each layer to adjust its importance and influence:

$$
I=\sum_{k=1}^{l} \alpha_{k} I_{k}, \quad \forall \alpha_{k} \geq 0 .
$$

However, selecting suitable layer weights $\left\{\alpha_{k}: 1 \leq k \leq l\right\}$ simultaneously is a formidable task. Based on Fisher criterion [40], we develop a criterion function to determine all the weights. It should be noted that the proposed criterion function is different from Fisher criterion, which can be formulated to minimize the trace ratio of the within-class scatter $S_{w}^{\prime}$ to the between-class scatter $S_{b}^{\prime}$, and simultaneously embed nonnegativity constraint on the weights. Let $I^{i, j}$ be the small-scale illumination-robust feature of the $j$ th sample from class $i$, and $m^{i}$ denotes the mean feature of the $i$ th class. Then the within-class scatter $S_{w}^{\prime}$ can be defined as

$$
S_{w}^{\prime}=\sum_{i=1}^{c} \sum_{j=1}^{n_{i}}\left(I^{i, j}-m^{i}\right)\left(I^{i, j}-m^{i}\right)^{T},
$$
class.

Let $I_{k}^{i, j}$ be the $k$ th layer of the small-scale illumination-robust feature $I^{i, j}$, and denote $m_{k}^{i}$ as the $k$ th layer of the mean feature $m^{i}$. Assigning a weight to each 
layer, $I^{i, j}$ and $m^{i}$ can be represented as $I^{i, j}=\sum_{k=1}^{l} \alpha_{k} I_{k}^{i, j}$ and $m^{i}=\sum_{k=1}^{l} \alpha_{k} m_{k}^{i}$, respectively. Substituting them into (7), we get

$$
\begin{aligned}
S_{w}^{\prime} & =\sum_{i=1}^{c} \sum_{j=1}^{n_{i}}\left(\sum_{k=1}^{l} \alpha_{k} I_{k}^{i, j}-\sum_{k=1}^{l} \alpha_{k} m_{k}^{i}\right)\left(\sum_{k=1}^{l} \alpha_{k} I_{k}^{i, j}-\sum_{k=1}^{l} \alpha_{k} m_{k}^{i}\right)^{T} \\
& =\sum_{i=1}^{c} \sum_{j=1}^{n_{i}}\left(\sum_{k=1}^{l} \alpha_{k}\left(I_{k}^{i, j}-m_{k}^{i}\right)\right)\left(\sum_{k=1}^{l} \alpha_{k}\left(I_{k}^{i, j}-m_{k}^{i}\right)\right)^{T} .
\end{aligned}
$$

In order to obtain a simpler form, we calculate the vector $A_{k}^{i, j}=I_{k}^{i, j}-m_{k}^{i}$, and denote $\boldsymbol{\alpha}=\left[\alpha_{1}, \alpha_{2}, \cdots, \alpha_{l}\right]^{T}$. Inserting them into (8) deduces:

$$
S_{w}^{\prime}=\sum_{i=1}^{c} \sum_{j=1}^{n_{i}}\left(A^{i, j}\right) \boldsymbol{\alpha} \boldsymbol{\alpha}^{T}\left(A^{i, j}\right)^{T},
$$

where matrix $A^{i, j}=\left[A_{1}^{i, j}, A_{2}^{i, j}, \cdots, A_{l}^{i, j}\right]$.

Similarly, let $m$ be the total mean feature of the sample set, and denote $m_{k}$ as the $k$ th layer of the total mean feature $m$. Then $m$ can be represented as $m=$ $\sum_{k=1}^{l} \alpha_{k} m_{k}$. Therefore, we can define the between-class scatter $S_{b}^{\prime}$ as follows:

$$
\begin{aligned}
S_{b}^{\prime} & =\sum_{i=1}^{c} n_{i}\left(m^{i}-m\right)\left(m^{i}-m\right)^{T} \\
& =\sum_{i=1}^{c} n_{i}\left(\sum_{k=1}^{l} \alpha_{k}\left(m_{k}^{i}-m_{k}\right)\right)\left(\sum_{k=1}^{l} \alpha_{k}\left(m_{k}^{i}-m_{k}\right)\right)^{T} \\
& =\sum_{i=1}^{c} n_{i}\left(B^{i}\right) \boldsymbol{\alpha} \boldsymbol{\alpha}^{T}\left(B^{i}\right)^{T}
\end{aligned}
$$

214 where matrix $B^{i}=\left[B_{1}^{i}, B_{2}^{i}, \cdots, B_{l}^{i}\right]$, and the vector $B_{k}^{i}=m_{k}^{i}-m_{k}(k=$ $2151,2, \cdots, l)$. 


\subsection{Discriminant filter learning}

After face image multi-layer decomposition, the small-scale illumination-robust component in each layer contains not only beneficial facial features, but also some detrimental features (noise) [21]. Such bad features would have a negative effect for face recognition. Similarly to [27] [41], we propose a discriminant filter learning method to deal with this problem. Given a reconstructed multi-layer weighted illumination-robust face image $I$, its filtered image is denoted as $f(I)$.

Under linear assumption, suppose the discriminant filter matrix to be $W$, and the filtered image can be represented as $f(I)=W^{T} I$. With the learned discriminant filter, the noise existed in the reconstructed illumination-robust face can be removed and the statistical discriminative ability is improved for face recognition under poor lighting conditions. By appropriate formulation, this problem can also be formulated as minimizing the trace ratio of the within-class scatter $S_{w}^{\prime \prime}$ to the between-class scatter $S_{b}^{\prime \prime}$, which are represented as follows:

$$
\begin{aligned}
S_{w}^{\prime \prime} & =\sum_{i=1}^{c} \sum_{j=1}^{n_{i}}\left(f(I)^{i, j}-f(m)^{i}\right)\left(f(I)^{i, j}-f(m)^{i}\right)^{T} \\
& =\sum_{i=1}^{c} \sum_{j=1}^{n_{i}} W^{T}\left(I^{i, j}-m^{i}\right)\left(I^{i, j}-m^{i}\right)^{T} W \\
S_{b}^{\prime \prime} & =\sum_{i=1}^{c} n_{i}\left(f(m)^{i}-f(m)\right)\left(f(m)^{i}-f(m)\right)^{T} \\
& =\sum_{i=1}^{c} n_{i} W^{T}\left(m^{i}-m\right)\left(m^{i}-m\right)^{T} W
\end{aligned}
$$

where $f(I)^{i, j}$ is filtered image for the $j$ th sample of class $i, f(m)^{i}$ is the mean filtered image of the $i$ th class, and $f(m)$ is the total mean filtered image of the sample set. 
By combining (9)-(12), the within-class scatter $S_{w}$ and the between-class scatter $S_{b}$ can be represented as follows:

$$
\begin{gathered}
S_{w}=\sum_{i=1}^{c} \sum_{j=1}^{n_{i}} W^{T}\left(A^{i, j}\right) \boldsymbol{\alpha} \boldsymbol{\alpha}^{T}\left(A^{i, j}\right)^{T} W \\
S_{b}=\sum_{i=1}^{c} n_{i} W^{T}\left(B^{i}\right) \boldsymbol{\alpha} \boldsymbol{\alpha}^{T}\left(B^{i}\right)^{T} W .
\end{gathered}
$$

According to the Fisher criterion, smaller within-class scatter and larger betweenclass scatter will be to better recognition results in an average sense. Therefore, we can define the constrained optimization problem for DMI as follows:

$$
\min _{W, \boldsymbol{\alpha}} \operatorname{Tr}\left(S_{w}\right)
$$

$$
\text { s.t. } \operatorname{Tr}\left(S_{b}\right)=1 \text { and } \boldsymbol{\alpha} \geq 0 \text {. }
$$

\subsection{Optimization algorithm}

Since it is difficult to directly solve the optimization problem in (15), we instead utilize an iterative, two-step strategy to alternately optimize $W$ and $\alpha$. At each iteration, one of the variables $W$ and $\alpha$ is fixed, while the other is updated, and then the roles of $W$ and $\alpha$ are exchanged. Iterations are repeated until convergence or the maximum number of iterations is reached.

On optimizing $W$. We could first assume that each layer feature is the same importance, and initialize the weight vector $\boldsymbol{\alpha}=[1,1, \cdots, 1]^{T}$. The optimization problem in (15) is then reduced to

$$
\min _{W} \operatorname{Tr}\left(W^{T} S_{w}^{\prime} W\right)
$$


where

$$
\begin{gathered}
S_{w}^{\prime}=\sum_{i=1}^{c} \sum_{j=1}^{n_{i}}\left(A^{i, j}\right) \boldsymbol{\alpha} \boldsymbol{\alpha}^{T}\left(A^{i, j}\right)^{T}, \\
S_{b}^{\prime}=\sum_{i=1}^{c} n_{i}\left(B^{i}\right) \boldsymbol{\alpha} \boldsymbol{\alpha}^{T}\left(B^{i}\right)^{T} .
\end{gathered}
$$

Intuitively, this optimization problem in (16) can be reduced to a trace ratio problem:

$$
\min _{W} \frac{\operatorname{Tr}\left(W^{T} S_{w}^{\prime} W\right)}{\operatorname{Tr}\left(W^{T} S_{b}^{\prime} W\right)}
$$

Consequently, according to [42], [43] and [44], the optimization problem can be transformed into solving the generalized eigenvalue problem. The columns of the optimal $W^{*}=\left[w_{1}, w_{2}, \cdots, w_{c-1}\right]$ are the eigenvectors with the $c-1$ smallest eigenvalues in

$$
S_{w}^{\prime} w=\lambda S_{b}^{\prime} w
$$

On optimizing $\alpha$. Once the matrix $W$ is updated, we could fix $W$ and use the property $\beta^{T} \beta=\operatorname{trace}\left(\beta \beta^{T}\right)$ for a column vector $\beta$, the optimization problem in (15) is reduced to

$$
\min _{\boldsymbol{\alpha}} \boldsymbol{\alpha}^{T} \tilde{S}_{w} \boldsymbol{\alpha}
$$

$$
\text { s.t. } \boldsymbol{\alpha}^{T} \tilde{S}_{b} \boldsymbol{\alpha}=1 \text { and } \boldsymbol{\alpha} \geq 0 \text {, }
$$

where

$$
\begin{aligned}
& \tilde{S}_{w}=\sum_{i=1}^{c} \sum_{j=1}^{n_{i}}\left(A^{i, j}\right)^{T} W W^{T}\left(A^{i, j}\right), \\
& \tilde{S}_{b}=\sum_{i=1}^{c} \sum_{j=1}^{n_{i}} n_{i}\left(B^{i}\right)^{T} W W^{T}\left(B^{i}\right) .
\end{aligned}
$$

The additional constraints $\alpha \geq 0$ cause that the optimization problem can not be formulated as a generalized eigenvalue problem. It becomes a non-convex 
quadratically constrained quadratic programming problem which is known to be hard to solve. However, the non-convex problem can be relaxed to a convex program by adding an auxiliary matrix $C=\boldsymbol{\alpha} \boldsymbol{\alpha}^{T}$ of size $l \times l$. The problem (21) can then be posed as:

$$
\begin{gathered}
\min _{\boldsymbol{\alpha}, C} \operatorname{trace}\left(\tilde{S}_{w} C\right) \\
\text { s.t. }\left\{\begin{array}{c}
\operatorname{trace}\left(\tilde{S}_{b} C\right)=1 \\
\left(\begin{array}{cc}
1 & \boldsymbol{\alpha}^{T} \\
\boldsymbol{\alpha} & C
\end{array}\right) \succeq 0 \\
\mathbf{e}_{k}^{T} \boldsymbol{\alpha} \geq 0, \quad k=1,2, \cdots, l
\end{array}\right.
\end{gathered}
$$

where $\mathbf{e}_{k}$ is a column vector whose elements are 0 except that its $k$ th element is 1 . This is a semi-definite programming (SDP) relaxation of the non-convex problem, which can be efficiently solved by SDP (Refer to [45] [42] for the details).

The whole algorithm of DMI is illustrated in Algorithm 2.

\subsection{Comparison Analysis of LTV, S\&L(LOG-DCT) and DMI}

Considering that LTV [16] and S\&L(LOG-DCT) [36] serve as the basis of our the proposed DMI, we take a comparison analysis of LTV, S\&L(LOG-DCT) and DMI.

1) Connections to LTV and $S \& L(L O G-D C T)$ : LTV takes advantage of the TV$L^{1}$ model to decompose a face image into small-scale component and large-scale component in logarithm domain, which utilizes the edge-preserving capability of total variation model. Both S\&L(LOG-DCT) and DMI also use the multiscale decomposition capability of the TV- $L^{1}$ model. Moreover, DMI, LTV, and S\&L(LOG-DCT) are based on the same idea that the small-scale component and 


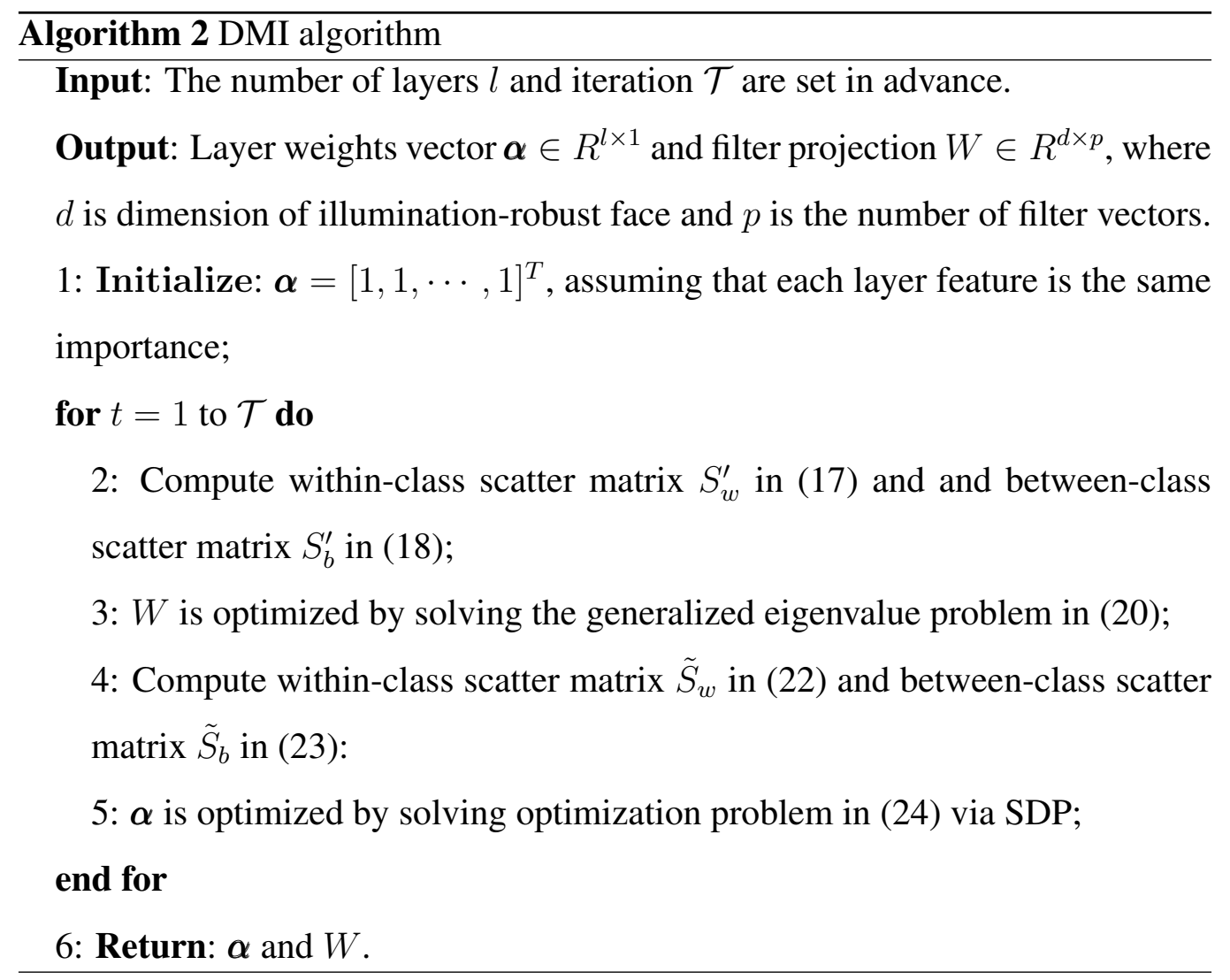

large-scale component can be separated from real image based on reflectance model.

2) Comparison Analysis: LTV only regards the small-scale component as facial feature for recognition, but large-scale component is ignored. However, S\&L(LOG-DCT) finds that both the small-scale component and large-scale component are important for recognition, so S\&L(LOG-DCT) performs a thresholdaverage filtering on the small-scale component and uses the LOG-DCT algorithm to preprocess the large-scale component. Finally, the normalized image is reconstructed by combining the smoothed small-scale component and the normalized large-scale component. However, it is difficult to separate the extrinsic illumi- 
nation variations and intrinsic facial structures from the large-scale component, and it is not effective to remove the detrimental features (noise, etc.) from the reconstructed face image, which may lead LTV and S\&L(LOG-DCT) methods to being unrobust to different lighting conditions. However, as can be seen in section 2.2, experiments show that the large-scale component contains a lot useful information for face recognition, and these information can be further extracted via the decomposition capability of the TV- $L^{1}$ model. Therefore, DMI decomposes the large-scale component into multi-layer small-scale illumination-robust features as a linear combination, and then a weight is assigned to each layer to adjust its importance and influence. Finally, the detrimental features can be removed from the reconstructed illumination-robust face via a discriminant filter learning method. The results provide an intuitionistic view that DMI is more robust to different lighting conditions than LTV and S\&L(LOG-DCT), especially in difficult lighting conditions. Comparisons between schemes of LTV, S\&L(LOG-DCT) and DMI are shown in Figure. 1.

\section{Experimental results}

The purpose of the presented experiment is to verify the following two points: i) the large-scale component contains useful information for face recognition, which can be further extracted, instead of discarded; ii) discriminant filter learning is effective to remove the noise from the reconstructed small-scale illuminationrobust face.

We compare our DMI with some of state-of-the-art illumination-treating-based and feature learning-based methods: LOG-DCT [17], LTV [16], Gradf [18], LBP [25], Webf [20], S\&L [36], LTP [26], DFD [27], MOST [28] and SVD face (SF) 
[22]. The four databases: Extended Yale B [46], Face Recognition Grand Challenge (FRGC) v2 [47], CAS-PEAL-R1 [48] and Oulu-CASIA-NIR-VIS database [49] are used to demonstrate the performance of different methods.

\subsection{Databases}

1) Extended Yale B: The Extended Yale B [46] consists of 2414 frontal-face images of 38 subjects. These images are divided into 5 Subsets according to their lighting conditions (the angle between the light source direction and the central camera axis). In our experiments, the cropped $192 \times 168$ face images are captured under various laboratory-controlled lighting conditions and different facial expressions, and each image is further downsampled to $96 \times 84$ pixels (except for LBP and SF). We select the Subset 1 for training, and the other 4 Subsets for testing, respectively. Figure. 3 shows some cropped images on this database.

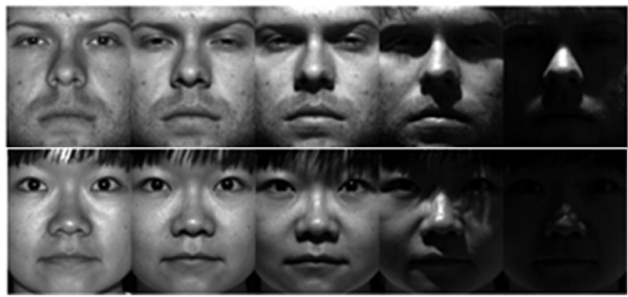

Figure 3: Cropped face images of two subjects from subsets 1-5 of the Extended Yale B database.

2) FRGC-v2: FRGC-v2 [47] is a large data set, which consists of 50,000 images of 625 subjects. These images were obtained in different period, under controlled and uncontrolled variations (illumination, expression, occlusion, etc.). In our experiment, we take a subset with uncontrolled variations, which were obtained in large lighting variations (hallways, atriums, outside, etc.), ageing and image blur. It contains 1375 images of 275 subjects and 5 images for each. These 


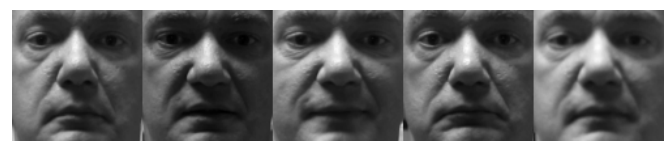

Figure 4: Cropped face images of the FRGC database.

are repeated 10 times. Figure. 5 shows some cropped images on this database.

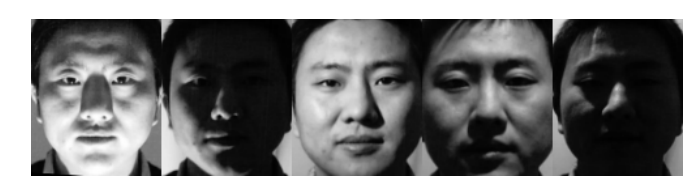

Figure 5: Cropped face images of the CAS-PEAL-R1 database. ther downsampled to $50 \times 50$ pixels (except for LBP and SF). In each recognition, 3 images per subject are selected for training and the left 2 for testing. There are $C_{5}^{3}=10$ turns of recognition in all, where $C_{b}^{a}$ denotes the combinatorial number of choosing $a$ elements from $b$ elements. Figure. 4 shows some cropped images on this database.

3) CAS-PEAL-R1: The CAS-PEAL-R1 [48] face database consists of 30900 images of 1040 subjects (595 males and 445 females). These images were obtained in different variations, including pose, expression, accessory, and lighting. In our experiment, we take a subset with different lighting variations, which contains 1404 images of 156 subjects and 9 images for each subject. All the images are cropped to $120 \times 100$ size, and each image is further downsampled to $60 \times 50$ pixels (except for LBP and SF). In each recognition, we randomly select 7 samples per subject for training, and the left 2 samples for testing. The experiments
4) Oulu-CASIA-NIR-VIS: The Oulu-CASIA-NIR-VIS database [49] contains

images are cropped and resized with dimension $100 \times 100$ and each image is fur- 
videos with six different illuminations and expressions from 80 subjects between 23 to 58 years old captured with two imaging systems. These video sequences consist of about 80000 frontal facial images from 80 subjects $(73.8 \%$ of them are males).

In our experiment, we select a subset of the database that consists $1440 \mathrm{im}$ ages of 80 subjects and 18 images for each subject. Each subject has two sessions of images and 9 images for each session. The first session contains NIR (Near Infrared) images under three different illumination conditions: normal illumination, weak illumination (only computer display is on), dark illumination (all lights are off). The second session contains VIS (Visible Light) images under the same illumination conditions as the first session. Thus, some of them are blurry and low-resolution images. All the images are cropped to $80 \times 70$ size, and each image is further downsampled to $40 \times 35$ pixels (except for LBP and SF). In each recognition, we randomly select half of the images for training and the remainder for testing. Some images are shown in Figure. 6.

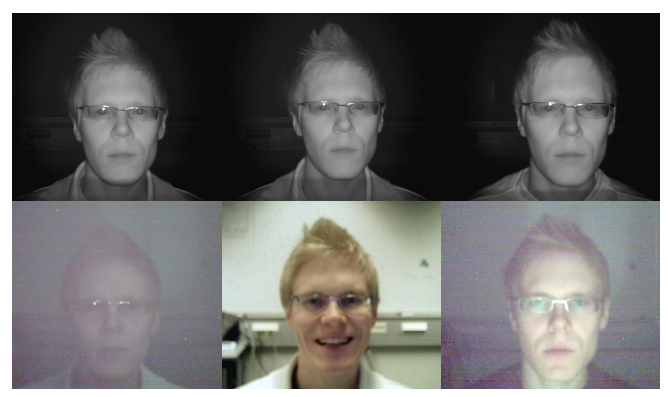

Figure 6: Some images of one subject in Oulu-CASIA-NIR-VIS database. The first row contains NIR (Near Infrared) images under three different illumination conditions: dark illumination, normal illumination, weak illumination. The second row contains VIS (Visible Light) images under three different illumination conditions: dark illumination, normal illumination, weak illumination. 
Table 2: Mean recognition rates of DMI with different $l$ on the Extended Yale B database (\%).

\begin{tabular}{cccccc}
\hline Method & Subset 2 & Subset 3 & Subset 4 & Subset 5 & Total \\
\hline $\operatorname{DMI}(l=4)$ & 100 & 99.12 & 96.43 & 97.37 & 97.82 \\
$\operatorname{DMI}(l=5)$ & 100 & 99.12 & 96.99 & 97.74 & 97.98 \\
$\operatorname{DMI}(l=6)$ & 100 & 99.12 & 96.99 & 97.37 & 97.98 \\
$\operatorname{DMI}(l=7)$ & 100 & 98.03 & 96.24 & 97.74 & 97.93 \\
$\operatorname{DMI}(l=8)$ & 100 & 99.12 & 96.24 & 97.56 & 97.87 \\
\hline
\end{tabular}

Table 3: Mean recognition rates of DMI with different $l$ on the FRGC database $(\%)$.

\begin{tabular}{cccccc}
\hline Method & $\operatorname{DMI}(l=4)$ & $\operatorname{DMI}(l=5)$ & $\operatorname{DMI}(l=6)$ & $\operatorname{DMI}(l=7)$ & $\operatorname{DMI}(l=8)$ \\
\hline Mean & 82.41 & 82.57 & 82.22 & 81.91 & 81.93 \\
\hline
\end{tabular}

\subsection{Robustness analysis and discussion}

In this section, we first analyse the robustness of our weight-selection mechanism for the proposed DMI model. Then the FRGC database is used to verify the robustness of DMI against noise. In addition, we discuss the effectiveness of layer weights and discriminant filter individually.

1) Robustness analysis to different number of layers $l$ : The selection of the number of layers $l$ must be appropriate. If the layer size $l$ is too small, the useful information will not be effectively extracted from the large-scale component. If the layer size $l$ is too large, it will lead to high computational complexity. Considering these, we set $l=4,5,6,7,8$. For each value of $l$, the controlled Extended Yale B database and uncontrolled FRGC database are used to demonstrate the robustness to different number of layers $l$. Following the recognition protocol in section 3.1, the experimental results of DMI with different number of layers $l$ are shown 
Table 4: Recognition rates (Mean \pm STD) and descent rates (DR) for different methods against noise on the FRGC database (\%).

\begin{tabular}{ccccc}
\hline \multirow{2}{*}{ Method } & \multicolumn{2}{c}{$\sigma=0.001$} & \multicolumn{2}{c}{$\sigma=0.01$} \\
& Mean \pm STD & DR & Mean \pm STD & DR \\
\hline LOG-DCT & $64.45 \pm 1.6480$ & 8.15 & $34.27 \pm 1.6054$ & 49.95 \\
Gradf & $67.06 \pm 3.2202$ & 9.82 & $57.32 \pm 2.4097$ & 22.92 \\
LTV & $57.35 \pm 2.0669$ & 8.15 & $17.73 \pm 1.3546$ & 67.30 \\
S\&L & $62.22 \pm 2.1322$ & $\mathbf{2 . 3 7}$ & $49.91 \pm 1.4140$ & 21.69 \\
LBP & $11.24 \pm 2.7159$ & 20.41 & $0.57 \pm 0.1520$ & 99.22 \\
Webf & $63.01 \pm 2.2505$ & 5.19 & $51.40 \pm 2.3892$ & 22.66 \\
DMI & $\mathbf{8 0 . 3 8} \pm \mathbf{1 . 7 7 3 2}$ & $\mathbf{2 . 6 5}$ & $\mathbf{7 2 . 4 4} \pm \mathbf{1 . 6 7 8 6}$ & $\mathbf{1 2 . 2 7}$ \\
\hline
\end{tabular}

in Table 2 and Table 3. As can be seen, the difference of the recognition rates of different layer size $l$ is not greater than $1 \%$ on the same subset of the Extended Yale $\mathrm{B}$ and the mean recognition rates are around $82 \%$ on the FRGC database. All these results show that our weight-selection mechanism is robust to a small change of layer size $l$.

2) Robustness analysis to different levels of noise: We evaluate the robustness of the proposed method against noise. Similarly to [50] [51], we adopt the common way of adding white Gaussian noise to the face images of the testing set but not the training set on the FRGC database. To intuitively compare the robustness of the different methods against noise, a descent rate is defined as follows:

$$
D R=\frac{r r-r r^{\prime}}{r r} \times 100 \%
$$

where $r r$ denotes the recognition rate of the method without noise, and $r r^{\prime}$ is the recognition rate with the white Gaussian noise. The recognition protocol is used 
Table 5: The effectiveness comparison of layer weights and discriminant filter on the Extended Yale B database (\%).

\begin{tabular}{cccccc}
\hline Method & Subset 2 & Subset 3 & Subset 4 & Subset 5 & Total \\
\hline LTV & 99.12 & 96.71 & 86.28 & 89.66 & 90.69 \\
S\&L & $\mathbf{1 0 0}$ & $\mathbf{9 9 . 1 2}$ & 87.97 & 87.03 & 93.07 \\
DMI $^{\alpha}$ & $\mathbf{1 0 0}$ & 97.81 & 96.43 & 95.30 & 97.72 \\
DMI $^{W}$ & $\mathbf{1 0 0}$ & 97.81 & 95.68 & 96.05 & 97.27 \\
DMI & $\mathbf{1 0 0}$ & $\mathbf{9 9 . 1 2}$ & $\mathbf{9 6 . 9 9}$ & $\mathbf{9 7 . 7 4}$ & $\mathbf{9 7 . 9 8}$ \\
\hline
\end{tabular}

as described before, and the experimental results for different methods with different levels of noise (The mean of Gaussian noise is zero and the variance $\sigma$ is set to 0.001 and 0.01 , respectively.) are shown in Table 4. As can be seen, the recognition rates of these methods decrease with the increasing of level of Gaussian noise. However, DMI, due to the use of discriminant filter, has the highest recognition rate and the lowest descent rate. It is obvious that the robustness of DMI against noise is notable as it outperforms the other methods for every level of noise.

3) Impact analysis of layer weights and discriminant filter: We investigate the effectiveness of layer weights and discriminant filter individually. First, we only apply the weight-selection mechanism without discriminant filter, denoted as $\mathrm{DMI}^{\alpha}$. In this case, the discriminant filter matrix is set to be $W=E$, where $E$ is the identity matrix. Second, we apply the discriminant filter without weightselection mechanism, denoted as $\mathrm{DMI}^{W}$. In this case, the layer weights $\alpha$ is set to be $\boldsymbol{\alpha}=[1,1, \cdots, 1]^{T}$ so that each layer illumination-invariant feature is treated equally. LTV and S\&L(LOG-DCT) are compared with our method. The compared 


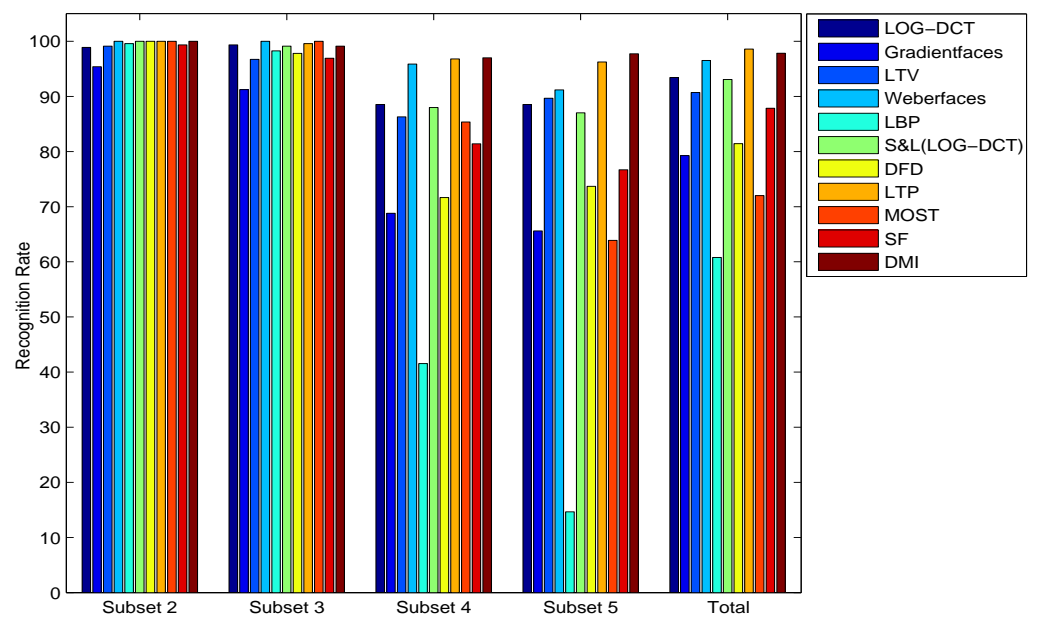

Figure 7: Recognition rates for different methods on Extended Yale B database.

results on Extended Yale B database are given in Table 5. As can be seen, both layer weights and discriminant filter help to improve the face recognition performance, compared with LTV and S\&L(LOG-DCT), especially in extremely harsh lighting conditions(Subsets 4 and 5). The combination of them further enhances the face recognition rate, indicating that the proposed DMI is effective for face recognition under different lighting conditions.

\subsection{Comparisons between DMI and other methods}

In this section, compared results between DMI and other methods on four databases are given.

1) Compared results on the Extended Yale B database: Following the recognition protocol in section 3.1, recognition rates for different methods on the Extended Yale B database are summarized in Table 6 and Figure. 7. According to the results, we can have the following conclusions:

- The superiority of DMI on Subset 2 and Subset 3 is not obvious, since 
Table 6: Mean recognition rates for different methods on the Extended Yale B database (\%).

\begin{tabular}{cccccc}
\hline Method & Subset 2 & Subset 3 & Subset 4 & Subset 5 & Total \\
\hline LOG-DCT & 98.9 & 99.34 & 88.53 & 88.53 & 93.42 \\
Gradientfaces & 95.39 & 91.23 & 68.8 & 65.6 & 79.25 \\
LTV & 99.12 & 96.71 & 86.28 & 89.66 & 90.69 \\
Weberfaces & $\mathbf{1 0 0}$ & $\mathbf{1 0 0}$ & 95.86 & 91.17 & 96.51 \\
LBP & 99.56 & 98.25 & 41.54 & 14.66 & 60.78 \\
LTP & $\mathbf{1 0 0}$ & 99.56 & 96.80 & 96.24 & $\mathbf{9 8 . 5 8}$ \\
DFD & $\mathbf{1 0 0}$ & 97.81 & 71.64 & 73.68 & 81.43 \\
MOST & $\mathbf{1 0 0}$ & $\mathbf{1 0 0}$ & 85.36 & 63.90 & 72.01 \\
S\&L(LOG-DCT) & $\mathbf{1 0 0}$ & 99.12 & 87.97 & 87.03 & 93.07 \\
SF & 99.34 & 96.93 & 81.39 & 76.69 & 87.85 \\
DMI & $\mathbf{1 0 0}$ & 99.12 & $\mathbf{9 6 . 9 9}$ & $\mathbf{9 7 . 7 4}$ & 97.98 \\
\hline
\end{tabular}

all these methods achieve the high performance, and the recognition rates are over $91 \%$, some even reach up to $100 \%$. It owes to the good lighting conditions of Subset 2 and Subset 3 and is easy for face recognition.

- The superiority of DMI gets more significant when lighting conditions get harsher from Subset 4 to Subset 5. For instance, the recognition rate of LBP is only $41.54 \%$ on Subset 4 , DMI is about $55.5 \%$ higher than it and $10 \%$ higher than LTV, which indicates that DMI can effectively extract illumination-robust features.

- Some feature learning/representation-based methods such as DFD and MOST, are robust to handle face recognition against small illumination variations, 
expression or pose variations, but they are degraded seriously under bad lighting conditions. For instance, The recognition rates of DFD are only $71.64 \%$ and $73.68 \%$ on Subset 4 and Subset 5, respectively. However, the proposed DMI reaches up to $96.99 \%$ and $97.74 \%$, respectively. The main reason is that DFD and MOST lack specialized background knowledge and do not extract illumination-invariant features when illumination variation dominates the main variation.

- Figure. 7 shows that the performance of DMI is steadily over these of other methods, no matter when the lighting conditions of the testing samples are good (Subsets 2-3) or harsh (Subsets 4-5), while the performance of other methods are unstable, especially for LBP and Gradientfaces.

2) Compared results on the FRGC database: FRGC-v2 is an uncontrolled illumination variations database, which is close to real-world scenarios. Therefore, it is more difficult than the Extended Yale B database for face recognition. Following the recognition protocol in section 3.1, the results of different methods are summarized in Table 7 and plotted in Figure. 8. According to the results, we can find that:

- The performances of feature learning/representation-based methods such as LTP, MOST, DFD, LBP and DMI are much better than image decompositionbased methods such as LTV, LOG-DCT and S\&L(LOG-DCT), which demonstrates that feature learning/representation-based methods are effective to handle face recognition on the uncontrolled illumination variation database.

- Compared with image decomposition-based methods such as LTV, LOGDCT and S\&L(LOG-DCT), DMI obtains much better recognition accura- 
Table 7: Mean recognition rates for different methods on the FRGC database (\%).

\begin{tabular}{cccccc}
\hline Method & LOG-DCT & Gradf & LTV & Webf & LBP \\
\hline Mean & 68.47 & 74.36 & 62.44 & 66.46 & 72.13 \\
\hline S\&L & LTP & DFD & MOST & SF & DMI \\
\hline 63.73 & 75.80 & 77.42 & 82.29 & 62.95 & $\mathbf{8 2 . 5 7}$ \\
\hline
\end{tabular}

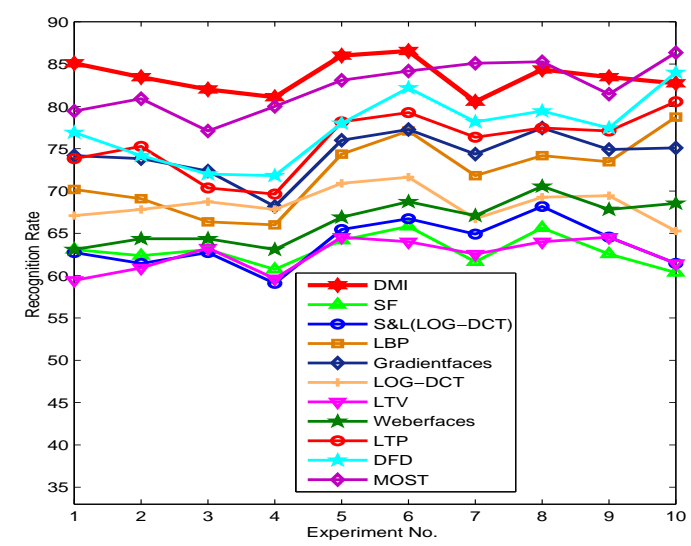

Figure 8: Recognition rates for different methods on FRGC database.

cies, which indicates that multi-layer decomposition strategy can effectively extract illumination-robust features in different layers.

- DMI obtains the best performance compared with these other feature learning/representation based methods such as LTP, MOST, DFD, LBP, and the recognition rate is $82.57 \%$. We think the superiority of DMI in dealing with uncontrolled face recognition is attributed to the discriminant filter learning.

3) Compared results on the CAS-PEAL-RI database: The lighting conditions on the CAS-PEAL-R1 database are somewhat less harsh than those of the Extended Yale B, but its sharp shadows make it harder for face recognition. Following 
Table 8: Mean recognition rates for different methods on the CAS-PEAL-R1 database (\%).

\begin{tabular}{cccccc}
\hline Method & LOG-DCT & Gradf & LTV & Webf & LBP \\
\hline Mean & 70.58 & 73.77 & 69.49 & 73.88 & 82.72 \\
\hline S\&L & LTP & DFD & MOST & SF & DMI \\
\hline 74.74 & 84.52 & 86.15 & 86.79 & 77.66 & $\mathbf{8 7 . 9 8}$ \\
\hline
\end{tabular}

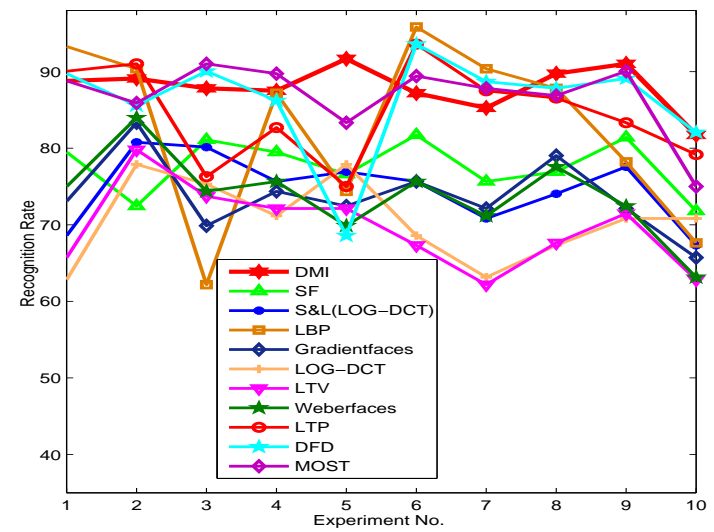

Figure 9: Recognition rates for different methods on the CAS-PEAL-R1 database.

the recognition protocol in section 3.1, the mean recognition rates and standard deviations for different methods are given in Table 8. As can we see, LOG-DCT, Gradientfaces, LTV, and Weberfaces all obtain low recognition rates of $70.58 \%$, $73.77 \%, 69.49 \%$ and $73.88 \%$, because these methods directly discard the largescale component, instead of extract useful information from it. LBP, LTP, DFD and MOST are feature extraction-based methods, whose performance are better than LOG-DCT, Gradientfaces, LTV, Weberfaces, S\&L(LOG-DCT) and SF, but they are still lower than DMI. From these results we have conclusion that discriminative weighed-layer illumination-robust features extraction model is effective to handle face recognition against illumination variations. 
Table 9: Mean recognition rates for different methods on the Oulu-CASIA-NIR-VIS database $(\%))$.

\begin{tabular}{cccccc}
\hline Method & LOG-DCT & Gradf & LTV & Webf & LBP \\
\hline Mean & 67.78 & 84.44 & 69.31 & 80.28 & 85.66 \\
\hline Method & S\&L & LTP & MOST & SF & DMI \\
\hline Mean & 72.64 & 85.28 & 86.53 & 83.33 & $\mathbf{8 6 . 9 4}$ \\
\hline
\end{tabular}

The recognition rates under the 10 different reference sets are shown in Figure. 9. we can find that DMI is always much better than most other state-of theart methods. Although LBP, LTP, DFD and MOST are slightly better than DMI on three of these reference sets, it is very unrobust and sensitive when the lighting conditions of reference sets are changing. For example, LBP only obtains recognition rates of $62.18 \%$ and $67.63 \%$ on the $3 \mathrm{rd}$ and 10 th reference sets, respectively. And our method can get recognition rates about $90 \%$ on all reference sets, it shows that DMI is robust and effective to reference sets under different lighting conditions.

4) Compared results on the Oulu-CASIA-NIR-VIS database: Oulu-CASIANIR-VIS is a video image database with different illumination and expression variations, which is close to real-world scenarios. Thus, it is more difficult than some other controlled illumination variations databases for recognition. According to the results in Table 9, we can find that:

- LTV, LOG-DCT and S\&L(LOG-DCT) get poor performance on the video image database, because they mainly use single-layer image decompositionbased methods to extract the illumination invariant features.

- The methods based on feature learning/representation such as MOST, D- 
MI and LTP significantly improve the performance compared with these traditional illumination-treating methods, which demonstrates the power of feature learning/representation on this database.

- The DMI obtains the best recognition performance among the compared methods, the recognition result is $86.94 \%$. It indicates that the DMI model is able to extract more discriminative and proper information for face recognition against different illumination conditions on video image scenario.

\section{Conclusion}

In this paper, we propose an illumination-robust feature extraction method DMI with discriminant face information for face recognition under poor lighting conditions. First, we use the decomposition capability of the TV- $L^{1}$ model to separate an original image into small-scale component and large-scale component. Some existing methods directly retain the small-scale component and remove the large-scale component (e.g. LOG-DCT and LTV). However, experimental results show that large-scale component contains some useful information for face recognition, which can be further extracted. Therefore, we further decompose the large-scale component into multi-layer small-scale features as a linear combination. Second, we assign a weight to adjust the importance and influence of each layer. Finally, we learn a discriminant filter to remove noise from the reconstructed illumination-robust face image. Experiments show that our weightselection mechanism is robust, and both layer weights and discriminant filter help to improve the face recognition performance. Moreover, the combination of them further enhances the face recognition rate, especially in extremely harsh lighting conditions. 
How to fuse multi-layer illumination-robust features to form a more compact and effective representation is a challenging task. Besides the scheme proposed in this paper, better ways of feature combination could be developed. For instance, could there concatenate directly the multi-layer illumination-robust features? If the answer is yes, how to overcome the huge dimensionality and computational problem. In addition, kernel methods have become popular in face recognition. Instead of linear combination, how to introduce nonlinear combination into the decision function? Our ongoing work will focus on these topics.

\section{References}

[1] M. Turk, A. Pentland, Eigenfaces for recognition, Journal of Cognitive Neuroscience 3 (1) (1991) 71-86.

[2] P. N. Belhumeur, J. P. Hespanha, D. Kriegman, Eigenfaces vs. fisherfaces: Recognition using class specific linear projection, IEEE Transactions on Pattern Analysis and Machine Intelligence 19 (7) (1997) 711-720.

[3] P. Comon, Independent component analysis, a new concept, Signal Processing 36 (3) (1994) 287-314.

[4] X. F. He, S. C. Yan, Y. X. Hu, P. Niyogi, H. J. Zhang, Face recognition using laplacianfaces, IEEE Transactions on Pattern Analysis and Machine Intelligence 27 (3) (2005) 328-340.

[5] Y. Xu, D. Zhang, J. Yang, J. Y. Yang, A two-phase test sample sparse representation method for use with face recognition, IEEE Transactions on Circuits and Systems for Video Technology 21 (9) (2011) 1255-1262. 
[6] R. He, W. S. Zheng, B. Hu, Maximum correntropy criterion for robust face recognition, IEEE Transactions on Pattern Analysis and Machine Intelligence 33 (8) (2010) 1561-1576.

[7] Z. R. Lai, D. Q. Dai, C. X. Ren, K. K. Huang, Discriminative and compact coding for robust face recognition, IEEE Transactions on Cybernetics 45 (9) (2015) 1900-1912.

[8] L. F. Wang, H. Y. Wu, C. H. Pan, Manifold regularized local sparse representation for face recognition, IEEE Transactions on Circuits and Systems for Video Technology 25 (4) (2015) 651-659.

[9] M. Yang, Z. Z. Feng, S. C. K. Shiu, L. Zhang, Fast and robust face recognition via coding residual map learning based adaptive masking, Pattern Recognition 47 (2) (2014) 535-543.

[10] S. G. Shan, W. Gao, B. Cao, D. B. Zhao, Illumination normalization for robust face recognition against varying lighting conditions, in: IEEE International Workshop on Analysis and Modeling of Faces and Gestures, 2003, pp. 157-164.

[11] S. Du, R. K. Ward, Adaptive region-based image enhancement method for robust face recognition under variable illumination conditions, IEEE Transactions on Circuits and Systems for Video Technology 20 (9) (2010) 11651175 .

[12] M. Savvides, B. V. Kumar, Illumination normalization using logarithm transforms for face authentication, in: International Conference on Audio- and Video-Based Biometric Person Authentication, 2003, pp. 549-556. 
[13] H. T. Wang, S. Z. Li, Y. S. Wang, Generalized quotient image, in: IEEE Conference on Computer Vision and Pattern Recognition, Vol. 2, 2004, pp. 498-505.

[14] K. N. Le, A mathematical approach to edge detection in hyperbolicdistributed and gaussian-distributed pixel-intensity images using hyperbolic and gaussian masks, Digital Signal Processing 21 (1) (2011) 162-181.

[15] P. P. Hegade, R. Nishanth, K. Manikantan, S. Ramachandran, Dwt-based face recognition using morphological edge detection as a pre-processing technique, 2013, pp. 1-6.

[16] T. Chen, W. Yin, X. S. Zhou, D. Comaniciu, T. S. Huang, Total variation models for variable lighting face recognition, IEEE Transactions on Pattern Analysis and Machine Intelligence 28 (9) (2006) 1519-1524.

[17] W. Chen, M. J. Er, S. Wu, Illumination compensation and normalization for robust face recognition using discrete cosine transform in logarithm domain, IEEE Transactions on Systems, Man, and Cybernetics, Part B: Cybernetics 36 (2) (2006) 458-466.

[18] T. P. Zhang, Y. Y. Tang, B. Fang, Z. W. Shang, X. Y. Liu, Face recognition under varying illumination using gradientfaces, IEEE Transactions on Image Processing 18 (11) (2009) 2599-2606.

[19] C. P. Chen, C. S. Chen, Intrinsic illumination subspace for lighting insensitive face recognition, IEEE Transactions on Cybernetics 42 (2) (2012) 422433. 
[20] B. Wang, W. F. Li, W. M. Yang, Q. M. Liao, Illumination normalization based on weber's law with application to face recognition, IEEE Signal Processing Letters 18 (8) (2011) 462-465.

[21] Z. R. Lai, D. Q. Dai, C. X. Ren, K. K. Huang, Multi-layer surface albedo for face recognition with reference images in bad lighting conditions, IEEE Transactions on Image Processing 23 (11) (2014) 4709 - 4723.

[22] W. Kim, S. Suh, W. Hwang, J. Han, Svd face: Illumination-invariant face representation, IEEE Signal Processing Letters 21 (11) (2014) 1336-1340.

[23] K. C. Kwak, W. Pedrycz, Face recognition using fuzzy integral and wavelet decomposition method, IEEE Transactions on Systems Man and Cybernetics Part B: Cybernetics 34 (4) (2004) 1666-1675.

[24] Y. Z. Goh, A. B. J. Teoh, M. K. O. Goh, Wavelet based illumination invariant preprocessing in face recognition, Journal of Electronic Imaging 18 (2) (2009) 421-425.

[25] T. Ahonen, A. Hadid, M. Pietikainen, Face description with local binary patterns: Application to face recognition, IEEE Transactions on Pattern Analysis and Machine Intelligence 28 (12) (2006) 2037-2041.

[26] X. Y. Tan, B. Triggs, Enhanced local texture feature sets for face recognition under difficult lighting conditions, IEEE Transactions on Image Processing 19 (6) (2010) 1635-1650.

[27] Z. Lei, M. Pietikainen, S. Z. Li, Learning discriminant face descriptor, IEEE Transactions on Pattern Analysis and Machine Intelligence 36 (2) (2014) 289-302. 
[28] C. X. Ren, D. Q. Dai, X. X. Li, Z. R. Lai, Band-reweighted gabor kernel embedding for face image representation and recognition, IEEE Transactions on Image Processing 23 (2) (2014) 725-740.

[29] T. Jabid, M. H. Kabir, O. Chae, Local directional pattern (ldp) for face recognition, in: IEEE International Conference on Consumer Electronics, 2010, pp. 329-330.

[30] F. J. Zhong, J. S. Zhang, Face recognition with enhanced local directional patterns, Neurocomputing 119 (2013) 375-384

[31] R. A. Ramirez, R. Castillo, O. Chae, Local directional number pattern for face analysis: Face and expression recognition, IEEE Transactions on Image Processing 22 (5) (2013) 1740-1752.

[32] M. R. Faraji, X. J. Qi, Face recognition under varying illumination based on adaptive homomorphic eight local directional patterns, IET Computer Vision 9 (3) (2015) 390-399.

[33] Y. Liu, F. Tang, Z. Zeng, Feature selection based on dependency margin, IEEE Transactions on Cybernetics 45 (6) (2014) 1209-1221.

[34] K. N. Le, K. P. Dabke, G. K. Egan, On mathematical derivations of auto-term functions and signal-to-noise ratios of the choi-williams, first- and nth-order hyperbolic kernels, Digital Signal Processing 16 (1) (2006) 84-104.

[35] L. I. Rudin, S. Osher, E. Fatemi, Nonlinear total variation based noise removal algorithms, 1992, pp. 259-268. 
[36] X. H. Xie, W. S. Zheng, J. H. Lai, P. C. Yuen, C. Y. Suen, Normalization of face illumination based on large-and small-scale features, IEEE Transactions on Image Processing 20 (7) (2011) 1807-1821.

[37] D. Strong, T. Chan, Edge-preserving and scale-dependent properties of total variation regularization, Inverse Problems 19 (6) (2003) 165-187.

[38] R. Ramamoorthi, P. Hanrahan, A signal-processing framework for inverse rendering, in: Annual Conference on Computer Graphics and Interactive Techniques, 2001, pp. 117-128.

[39] H. Han, S. G. Shan, X. L. Chen, S. H. Lao, W. Gao, Separability oriented preprocessing for illumination-insensitive face recognition, in: European Conference on Computer Vision, 2012, pp. 307-320.

[40] R. O. Duda, P. E. Hart, D. G. Stork, Pattern classification. 2nd, Edition. New York.

[41] L. C. Zhang, S. Wei, L. Qu, Kernel-based discriminant image filter learning: application in face recognition, in: SPIE/COS Photonics Asia, 2014, pp. 110 .

[42] Y. Y. Lin, T. L. Liu, C. S. Fuh, Multiple kernel learning for dimensionality reduction, IEEE Transactions on Pattern Analysis and Machine Intelligence 33 (6) (2011) 1147-1160.

[43] H. T. Chen, H. W. Chang, T. L. Liu, Local discriminant embedding and its variants, in: IEEE Conference on Computer Vision and Pattern Recognition, 2005, pp. 846-853. 
[44] H. Wang, S. C. Yan, D. Xu, X. O. Tang, T. Huang, Trace ratio vs. ratio trace for dimensionality reduction, in: IEEE Conference on Computer Vision and Pattern Recognition, 2007, pp. 1-8.

[45] L. Vandenberghe, S. Boydand, Semidefinite programming, SIAM Review 38 (1996) 49-95.

[46] A. S. Georghiades, P. N. Belhumeur, D. J. Kriegman, From few to many: Illumination cone models for face recognition under variable lighting and pose, IEEE Transactions on Pattern Analysis and Machine Intelligence 23 (6) (2001) 643-660.

[47] P. J. Phillips, P. J. Flynn, T. Scruggs, K. W. Bowyer, J. Chang, K. Hoffman, J. Marques, J. Min, W. Worek, Overview of the face recognition grand challenge, in: IEEE Conference on Computer vision and pattern recognition, 2005, pp. 947-954.

[48] W. Gao, B. Cao, S. G. Shan, X. L. Chen, D. L. Zhou, X. H. Zhang, D. B. Zhao, The cas-peal large-scale chinese face database and baseline evaluations, IEEE Transactions on Systems, Man and Cybernetics, Part A: Systems and Humans 38 (1) (2008) 149-161.

[49] G. Y. Zhao, X. H. Huang, M. Taini, S. Z. Li, M. Pietikainen, Facial expression recognition from near-infrared videos, Image and Vision Computing 29 (9) (2011) 607-619.

[50] W. Wang, L. Xu, A modified sparse representation method for facial expression recognition, Computational Intelligence and Neuroscience 2016 (3) (2016) 1-12. 
[51] R. S. Kathavarayan, M. Karuppasamy, Preserving global and local features for robust face recognition under various noisy environments, International Journal of Image Processing 3 (6) (2010) 328-340. 